\title{
PUEBLOS INDÍGENAS, INJUSTICIAS HISTÓRICAS Y PRESENTES: ENTREVISTA A TIMOTHY WALIGORE
}

Por Santiago Truccone Borgogno

(Universidad de Graz / Austria) santiagotruccone@gmail.com

Traducción: Romina Frontalini Rekers

(Universidad Nacional de Córdoba / Argentina) rominarekers@gmail.com

Recibido: $18 / 09 / 2018$

Aprobado: 30/10/2018

Timothy Waligore es actualmente Profesor asistente en Ciencia Politica y coordinador de Estudios Globales de la Universidad de Pace en Nueva York. Es profesor visitante regular de la Universidad de Graz en Austria donde es co-director (junto con Lukas Meyer) de un proyecto sobre rectificación y anulación (supersession) de injusticias históricas, financiado por el FWF (Fondo Austriaco para la Ciencia). Es Ph. D. en Ciencia Política por la Universidad de Columbia. Ha publicado en Politics, Philosophy \& Economics, Moral Philosophy and Politics, Public Reason: Journal of Moral and Political Philosophy, Analyze \& Kritik, The Nation y en The New Republic. Es co-editor, junto con Barbara Buckinx y Jonathan TrejoMathys, de Domination and Global Political Justice (Routledge 2015). Su investigación se centra en resolver reclamos basados en injusticias históricas, justiciar global y ciudadanía multicultural.

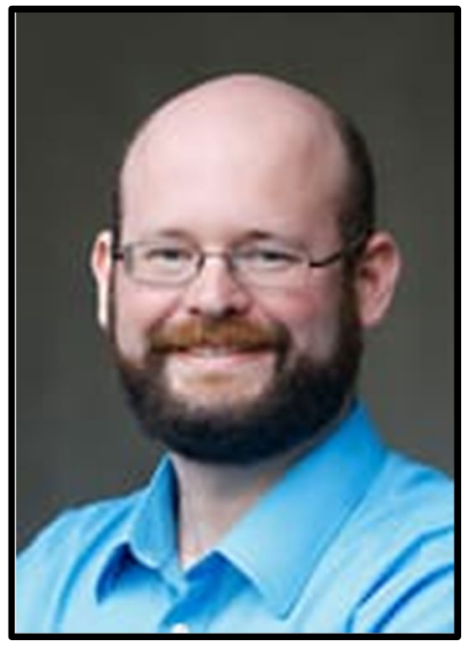

1. Una de nuestras mayores preocupaciones cuando pensamos en la justicia se refiere a su orientación temporal. ¿Por qué crees que esta característica es importante?

Algunas personas podrían estar interesadas en esto desde un punto de vista teórico más abstracto. A mí me interesa esta característica desde un punto de vista práctico: ¿cómo podrían justificarse o no las reparaciones? En relación con este problema, tres enfoques son comunes entre los teóricos políticos. En primer lugar, los enfoques prospectivos (forward-looking) pretenden aliviar las situaciones de desventaja del presente y del futuro, sin contar con una preocupación fundamental por rectificar las injusticias históricas. Este es un punto de vista muy popular y creo que es incorrecto. Este enfoque podría permitir la devolución de tierras y demás cosas a las personas históricamente desposeídas. Sin embargo, tales soluciones no capturan los males realmente cometidos. En segundo lugar, los enfoques retrospectivos (backward-looking) le otorgan una importancia moral independiente a la rectificación de males históricos, incluso si esto llegara a beneficiar a quienes están (o estarán) en las posiciones más favorecidas. Tengo simpatía por este enfoque, pero no creo que una versión no revisada tenga éxito. No podemos, por ejemplo, ignorar las necesidades actuales. En tercer lugar, los enfoques basados en la desventaja estructural se centran en que la injusticia continúa arraigada en el pasado y nos dicen cómo las desventajas estructurales tienden a persistir en el tiempo. Considero que este 
enfoque puede resolver muchos problemas importantes, pero, en última instancia, creo que está demasiado orientado al presente.

2. ¿Por qué crees que los eventos pasados pueden cambiar los requerimientos presentes de la justicia?

Esta es una pregunta difícil. Robert Nozick y otros libertarios con concepciones basadas en derechos tienen una respuesta directa. La justicia es el resultado de una serie de adquisiciones y transferencias justas. Para Nozick, la justicia no se fundamenta en patrones; no deberíamos aspirar a un patrón como el de "igualdad" o "a cada cual según su merecido". La justicia tiene una dimensión históricamente ineludible. Entonces, Nozick dice que, si algo fue tomado de modo incorrecto, debería devolverse. Aun así, para Nozick, el estándar de justicia sigue siendo el mismo antes y después de una injusticia. He argumentado que lo que públicamente se considera como injusticia puede cambiar como resultado de una injusticia pasada. El teórico liberal prospectivista, John Rawls, está preocupado por las bases sociales del autorespeto y por garantizar a ciudadanos iguales que se hará justicia. Bueno, para que pueda considerarse que se ha hecho justicia, debe ser visible que se ha hecho justicia. Así que, adaptando Rawls en función de sus propios fines, argumento que a raíz de una injusticia histórica puede ser más difícil asegurar a algunos grupos, tales como los nativos americanos y los afroamericanos, que se hará justicia. Por lo que, por ejemplo, aspirar a la igualdad entre los miembros promedio de diferentes grupos podría justificarse como una forma de asegurar a las personas que hay igualdad de oportunidades. Esto podría parecerse a las reparaciones históricas Nozickeanas, pero se fundamenta en las preocupaciones prospectivas de proporcionar las bases sociales para el autorespeto y la seguridad. Ese es un argumento que he usado. Creo que es bastante moderado, pero es interesante considerarlo y aquí me ha inspirado el trabajo de Charles Mills. Rawls es pensado como el ejemplo de los teóricos prospectivistas, aunque si estoy en lo cierto, es interesante pensar que la estructura de sus ideas podría (y debería) adaptarse en respuesta a las injusticias históricas. Creo que hay mucho trabajo interesante por hacer aquí y, realmente, solo hemos empezado a explorarlo.

3. Una gran parte de tu trabajo se ocupa de cuestiones indígenas. ¿Qué crees que deberíamos hacer sobre este asunto?

Me concentro principalmente en los Estados Unidos y Canadá, por lo que esas son las áreas con las que estoy más familiarizado. Mi punto de vista es que debe devolverse una gran cantidad de tierra y los pueblos indígenas deberían tener jurisdicción real sobre esas tierras. En términos más generales, mi enfoque supone que la tierra y la jurisdicción deben ser reconocidas y devueltas, mientras se consideren los límites fundados en principios que existen sobre esto. Un enfoque alternativo, del que no estoy a favor, considera que todas las personas, incluidos los pueblos indígenas, son simplemente miembros iguales de un estado unitario. Para este enfoque, lo que es importante es una justicia distributiva prospectiva. Sin embargo, es importante reconocer que las injusticias contra los pueblos indígenas no son solo históricas, sino que están en curso. Muchas de las decisiones de la Corte Suprema de EE. UU. en las últimas décadas han erosionado gradualmente la soberanía indígena, y eso debería revertirse.

\section{4. ¿Piensa que su respuesta anterior podría cambiar cuando consideramos lo que la justicia} requiere en países con grandes patrones de desigualdad o con altas tasas de pobreza?

Dije que debe haber límites fundados en principios sobre cuánto se puede devolver la tierra. Se podría argumentar que uno de estos límites es que todos deberían tener un nivel suficiente de bienestar (lo que se llama "suficiencia" en la filosofía liberal). Otros van más allá y dicen que todos deberían tener suficiente para no ser dominados por otros. Pero incluso con estas condiciones, hay o podría haber mucho espacio para los reclamos basados históricamente. También soy extremadamente desconfiado de la idea de que las tierras de los pueblos indígenas deban minarse por recursos que, supuestamente, ayudarán a los pobres. Primero, no creo que esto sea sostenible. Segundo, sospecho que esto no ayuda mucho a los pobres, beneficia mucho más a los ricos. Tercero, sospecho que hay mejores formas de ayudar a los pobres. Puede que no sean tan políticamente factibles porque afectan a los mejor 
acomodados, pero eso no es excusa. Los pueblos indígenas no deberían soportar el impacto de esto porque son un objetivo fácil sin tanto poder político. Cuarto, aquí hay una dimensión internacional o cosmopolita importante. En mi opinión los pueblos indígenas no son parte plena de un Estado soberano unitario y no están libres de problemas. Si uno piensa que los deberes de justicia distributiva son diferentes en el ámbito doméstico y global, entonces no está claro que los pueblos indígenas tengan las mismas responsabilidades que los ciudadanos regulares del país.

5. Una vez, cuando estaba haciendo una presentación sobre la famosa tesis de la supersession, una de los asistentes, una mujer cacique de los Comechingones, interrumpió mi exposición diciéndome que "estaba hablando como los abogados de los terratenientes extranjeros que querían quitarles sus tierras ancestrales". Le dije que, si pueblos indígenas sostenían lo que estaba presentando, entonces las tierras deberían ser entregadas a la tribu Comechingón. Sin embargo, ella me dijo algo que me dejó perplejo: "No es lo que usted está diciendo, es la forma en que habla". Creo que ella estaba diciendo que mi presentación era percibida como una especie de imposición. ¿Qué piensa de este episodio?

No puedo hablar de lo que ella estaba pensando, sobre todo porque no estaba allí y parecía querer ser escuchada con su propia voz. Pero esto me hace pensar en varias cosas que vale la pena considerar, cualquiera haya sido su intención. En primer lugar, incluso si su análisis sobre la supersession conduce a una conclusión favorable para un pueblo indígena en particular, los conceptos que utilizas para llegar a esa conclusión pueden tender a empujar en la otra dirección. Otros pueden haber usado esos conceptos en el pasado para llegar a conclusiones mucho menos favorables, y aún pueden hacerlo hoy. Hablando más concretamente en términos de lenguaje legal, en muchos contextos no todos piensan que el logro de los derechos sobre el papel es tan importante; si los derechos no pueden implementarse en la práctica, pueden valer poco o nada. Algunos grupos tienen una mayor capacidad para manipular el sistema legal por lo que, incluso si se puede presentar un argumento inteligente que reivindique los derechos indígenas, podría no significar nada en realidad. Y los pueblos indígenas pueden desconfiar de utilizar un lenguaje que ha sido utilizado históricamente para despojarlos de sus derechos. En segundo lugar, hay académicos indígenas, aunque los que tengo en mente no son de América del Sur, que piensan que las ideas indígenas deben ser una parte importante del diálogo. Poner las cosas en términos de filosofía política analítica liberal puede malinterpretar estas ideas. En Canadá, un pueblo indígena tuvo que ganar un juicio para que se reconociera su tradición oral como una forma de proporcionar pruebas del hecho de que estuvieron en su tierra por mucho tiempo. Pero incluso si los pueblos indígenas obtienen victorias en los tribunales, el sistema judicial canadiense y el sistema político tienden a afirmar que el autogobierno indígena y los reclamos de tierras deben reconciliarse con la soberanía de la Corona (Canadá todavía tiene a la Reina de Inglaterra como cabeza oficial de Estado). Entonces esto podría ser visto como una imposición colonial. Tercero, requiere mucho trabajo tratar de traducir las ideas indígenas al lenguaje del sistema legal dominante o al modo de argumentación filosófica, si esto es posible en toda su extensión. Obligar a los pueblos indígenas a hacer esto para que sean escuchados podría ser llamado imposición. Además, algunas personas pueden pensar que el uso de un determinado idioma es una imposición colonial, como usted mencionó, ya que privilegia cierto tipo de discurso. También hay otras posibilidades, por supuesto. Es importante tratar de hacerse consciente, en la medida de lo posible, de las concepciones de vida, modos de ser y modos de pensar indígenas, algo que aquellos de nosotros que trabajamos en estos temas, a menudo, no hacemos lo suficiente. 